\title{
GESTÃO DOS ESTOQUES NUMA CADEIA DE DISTRIBUIÇÃO COM SISTEMA DE REPOSIÇÃO AUTOMÁTICA E AMBIENTE COLABORATIVO
}

\author{
George Paulus Pereira Dias e Hugo Tsugunobu Yoshida Yoshizaki \\ Escola Politécnica da USP - Departamento de Engenharia de Produção \\ Av. Prof. Almeida Prado, trav.2, 128, $2^{\circ}$ andar, CEP 05508-900, São Paulo-SP
}

\section{RESUMO}

O foco do presente trabalho está na gestão de estoques em sistemas multicamadas. O modelo de simulação construído considera o desempenho histórico de uma cadeia de distribuição de medicamentos versus a política de gestão proposta.

Os objetivos principais do trabalho são: a avaliação quantitativa da política de cálculo de necessidades aplicada na gestão de sistemas multicamadas, a verificação da importância da cooperação entre os elos da cadeia de distribuição para gestão do fluxo de materiais e o estudo das curvas que representam o dilema 'nível de serviço' versus 'custo total da cadeia'.

Inicialmente, cada cenário simulado é preparado com a definição do 'período de aquecimento', 'horizonte de simulação' e 'número de réplicas' necessárias. Depois disso, é feito o delineamento de experimentos para identificar quais variáveis de decisão têm efeito significativo sobre o custo total da cadeia. Finalmente, é feita uma busca da parametrização de cada um dos cenários que apresente o melhor custo total da cadeia.

Os resultados da simulação mostraram que as práticas atualmente empregadas na gestão dos estoques das empresas podem ter seu desempenho melhorado com a utilização da política simulada na pesquisa. Nas simulações pode-se verificar a melhoria simultânea do nível de serviço e do nível de estoques da cadeia. Isso mostra que a política simulada mudou o dilema (trade-off) que interliga antagonicamente essas duas características de desempenho do sistema.

\begin{abstract}
This paper focuses on the inventory management for multi-echelon systems. The simulation model proposes a new inventory management policy and compares it to the historical performance of a medicine supply chain.

The main objectives of this research are: the quantitative analysis of the method used for the calculation of material requirements in multi-echelon systems; the verification of the importance of the cooperation between the components of the supply chain to the material flow management; and the analysis of the curves which represent the trade-off between 'service level' and 'total cost'.

Initially, each scenario was prepared with the definition of the 'warm-up' period, the simulation horizon and the amount of required replications. Secondly, the design of experiments (DOE) was done in order to determine which decision variables have influence on the supply chain total cost. Finally, each scenario was tested with many different parameters in order to find the lowest cost for the supply chain.

The simulation results have showed that the procedures currently applied for the inventory management can have their performance improved by the use of the policy proposed in this research. In the simulations, both the 'service level' and the 'total cost' have improved. In this manner, it can be said that the new policy has improved this trade-off.
\end{abstract}

\section{INTRODUÇÃO}

Os estoques estão presentes ao longo das cadeias de distribuição pelos mais diversos motivos. Ballou (1998) estima que o custo anual de manutenção de um item em estoque é de 20 a $40 \%$ do seu valor. Lee \& Billington (1992) apontam diversas oportunidades para a melhoria na gestão de estoques na cadeia e utilizam sempre a premissa de que o menor nível de estoques é obtido quando toda cadeia é considerada como um sistema único. 
Com o uso das práticas de cooperação e compartilhamento de informações, pretende-se estudar como o estabelecimento da política de estocagem influencia o dilema existente entre a disponibilidade de produtos (Nível de serviço) e os custos de estocagem (Nível de estoque) em dois estágios da cadeia.

Este trabalho tem como principal foco o gerenciamento da disponibilidade de produtos, através da política de gestão de estoque, numa cadeia de distribuição em que haja cooperação e compartilhamento de informações.

Os estoques na cadeia de distribuição são necessários para que o processo de produção e distribuição de um produto possa ocorrer. Na Tabela 1 estão apresentados a consolidação dos motivos pelos quais os estoque são necessários, bem como a classificação de cada tipo de estoque conforme Robeson et al. (1994).

Tabela 1 - Forças que tornam os estoques necessários (Robeson et al., 1994)

\begin{tabular}{|l|l|}
\hline Motivo do estoque & Tipo de estoque \\
\hline Incertezas & Estoque de segurança \\
\hline Produção/Transporte em lotes & Estoque de ciclo \\
\hline Tempo de transporte & Estoque em trânsito \\
\hline Tempo de processamento & Estoque em processo \\
\hline Sazonalidade & Estoques sazonais \\
\hline Variação na taxa de atividades & Estoque de antecipação \\
\hline Outros & Estoques especulativos \\
\hline
\end{tabular}

A gestão de estoques procura balancear, de um lado, um determinado nível de disponibilidade dos produtos e, do outro, os custos necessários para atingir esse nível de serviço. Os sistemas multicamadas são aqueles que possuem mais de um ponto de estocagem e cujos pontos de estocagem alimentam uns aos outros.

O estudo das redes de distribuição, o efeito chicote (também conhecido como efeito bullwhip ou efeito Forrester). Como descrito a seguir, o efeito chicote é um fenômeno importante no estudo das cadeias de distribuição porque tem conseqüências sobre a eficiência do fluxo de materiais nas cadeias de suprimento e distribuição.

Forrester (1958) marca o início do conceito de compartilhamento de informações na gestão da cadeia de suprimentos. Ele mostrou que as informações, assim como os pedidos, se propagam com maior volatilidade quando se caminha à montante na cadeia de distribuição (e/ou de suprimentos). Lee et al. (1997b) chamaram esse fenômeno de efeito "bullwhip" ou efeito chicote. A Figura 1 mostra um exemplo do efeito chicote, apresentado por Simchi-Levi (1998).

Lee et al. (1997) sugerem práticas que ataquem o efeito chicote em cada uma das causas apontadas por eles:

- Processamento das variações na demanda - a distorção da demanda surge devido à falta de visibilidade que os fornecedores e fabricantes têm do real consumo de seus produtos. Uma forma de reduzir esse aspecto é compartilhando as informações de consumo com as empresas que atuam na cadeia de distribuição. Mesmo assim, as diferentes metodologias de previsão que são utilizadas entre as empresas vão manter o efeito chicote. "Para eliminar o efeito chicote, podemos antever um único membro da cadeia realizando as atividades de previsão e compras para as outras empresas" (Lee et al., 1997, pp. 556). Lee et al. (1997) apontam ainda práticas como VMI (estoque gerenciado pelo fornecedor) e CRP (programas de reposição automática) como vetores que atuam no sentido da redução do efeito chicote. A eliminação de etapas na cadeia de distribuição e a redução dos tempos de ressuprimento também podem ser usadas na redução do efeito chicote.

- Racionamento (compras de prevenção à falta) - em situações em que há falta de produtos a tendência é que as empresas peçam quantidades maiores do que sua real necessidade. Nesse caso, a alocação da quantidade disponível para entrega pode ser feita de acordo com a participação histórica de mercado de cada cliente, e não segundo seus pedidos feitos no período de falta. O mesmo ocorre quando uma empresa procura proteger-se contra uma possível falta. Neste caso, a fim de evitar pedidos distorcidos, o fabricante deve compartilhar informações de estoque e produção.

- Formação de lotes de compra e de produção - as causas para a utilização dos lotes são os custos fixos de pedido, produção e transporte e a utilização de 'períodos de revisão dos estoques' sem que o fabricante tenha informações sobre o consumo de seu produto. Dessa forma, o combate ao efeito chicote se dá através da redução dos custos fixos de pedido como, por exemplo, a utilização dos 
sistemas automáticos de reposição sem a necessidade da emissão de pedidos por papel. Quanto à revisão periódica, seu efeito pode ser diminuído com a disponibilização da informação de consumo ao longo da cadeia de distribuição.

Variações de preço - as distorções no fluxo de materiais causadas pelas estratégias de variações no preço devem ser evitadas com a utilização de outras políticas comerciais como, por exemplo, a política de preço baixo todo dia (every day low price). Outra alternativa é a desvinculação contratual entre a compra e a entrega dos produtos. Assim uma grande compra para obtenção de descontos pode ser dividida em várias entregas em períodos futuros.

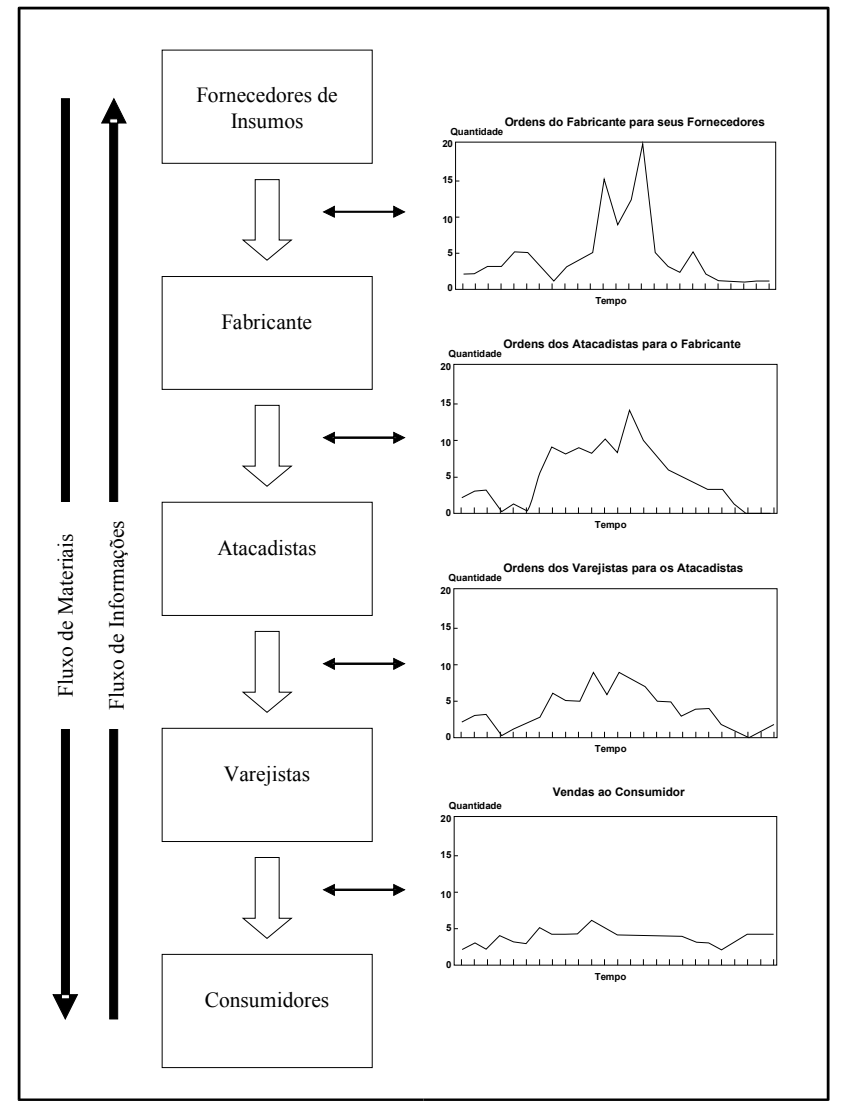

Figura 1 - Exemplo de efeito chicote (Simchi-Levi, 1998)

Os resultados obtidos nas pesquisas sobre a redução do efeito chicote podem ser classificadas em três tipos de iniciativas (vide Tabela 2):

a. A alteração do processo físico de produção, armazenagem e transporte dos materiais;

b. O compartilhamento de informações para o planejamento em cada etapa da cadeia logística;

c. O controle dos efeitos das políticas comerciais para que estas não induzam o fluxo da cadeia ao efeito chicote.

Tabela 2 - Indicações para redução do efeito chicote

\begin{tabular}{|l|l|l|l|}
\hline & Forrester (1958) & Lee et al.(1997) & Simchi-Levi et al. (2000) \\
\hline $\begin{array}{l}\text { Melhoria do processo } \\
\text { logístico }\end{array}$ & $\begin{array}{l}\text { - Agilização do } \\
\text { tratamento dos pedidos }\end{array}$ & $\begin{array}{l}\text { - Formação de lotes } \\
\text { de compra e de } \\
\text { produção }\end{array}$ & $\begin{array}{l}\text { - Redução do tempo de } \\
\text { ressuprimento }\end{array}$ \\
\hline $\begin{array}{l}\text { Compartilhamento de } \\
\text { informações }\end{array}$ & $\begin{array}{l}\text { - Melhora na qualidade } \\
\text { dos dados } \\
\text { - Ajuste dos estoques }\end{array}$ & $\begin{array}{l}\text { - Processamento } \\
\text { das variações na } \\
\text { demanda }\end{array}$ & $\begin{array}{l}\text {-Formação de parcerias } \\
\text { estratégicas } \\
\text {-Redução da incerteza }\end{array}$ \\
\hline $\begin{array}{l}\text { Redução do efeito de } \\
\text { políticas comerciais }\end{array}$ & $\begin{array}{l}\text { - Variações de } \\
\text { preço } \\
\text { - Racionamento }\end{array}$ & -Redução da variabilidade \\
\hline
\end{tabular}

METODOLOGIA DE PESQUISA 
Devido às dificuldades com relação ao desenvolvimento das técnicas analíticas para definição de políticas para gestão de estoques em sistemas multicamadas, a alternativa da simulação mostra-se como uma importante ferramenta para o tratamento do problema. De acordo com Ballou (1998) os estoques ao longo da cadeia de distribuição são raramente independentes entre si e a gestão dos estoques ao longo de toda cadeia torna-se mais importante do que a gestão de um ponto de estoque independente. Entretanto, problemas dessa natureza tornam-se muito complexos para serem tratados por modelos matemáticos analíticos. "O planejamento de estoques através de vários níveis (echelons) da cadeia de distribuição é usualmente feita com a ajuda de simulação por computador.” (Ballou, 1998, pp. 349).

"O maior beneficio das técnicas de simulação de estoques é sua capacidade de modelar uma ampla variedade de ambientes logísticos sem precisar de pressupostos simplificadores (...) A maior desvantagem das técnicas de simulação é sua limitação para buscar e identificar soluções ótimas (...). A simulação está se tornando mais popular com a tentativa das empresas em entender a dinâmica dos estoques nos canais logísticos" (Bowersox \& Closs, 1996, pp. 573). Modelos estocásticos de cadeias de suprimento são desenvolvidos para capturar complexidades de um problema multi-produto, multi-camadas, multinacional, multi-período de gestão. "Até mesmo problemas de pequenas proporções podem resultar em problemas de otimização complexos e que só podem ser eficientemente avaliados utilizando métodos de simulação" (Tayur et al., 1998).

Simchi-Levi et al. (2000) ressaltam que os modelos de simulação permitem análises apenas para uma situação específica que esteja sendo simulada e que modelos complexos podem exigir grande potencial computacional para executar as simulações. A alternativa sugerida é a utilização dos modelos analíticos para gerar uma solução macro do problema e em seguida a utilização da simulação para avaliar as soluções geradas na primeira fase.

É importante ressaltar que, apesar dos avanços relacionados à utilização das técnicas de busca em modelos de em simulações, este continua sendo um ponto fraco da simulação em relação à modelagem analítica de problemas. De acordo com Bowden \& Hall (1998), a associação das técnicas de busca a técnicas de simulação é uma prática recente e muitas melhorias ainda devem ser feitas nessa área da simulação.

A Figura 2 apresenta a metodologia utilizada para a construção e experimentação do modelo de simulação conforme Bateman et al. (1997)

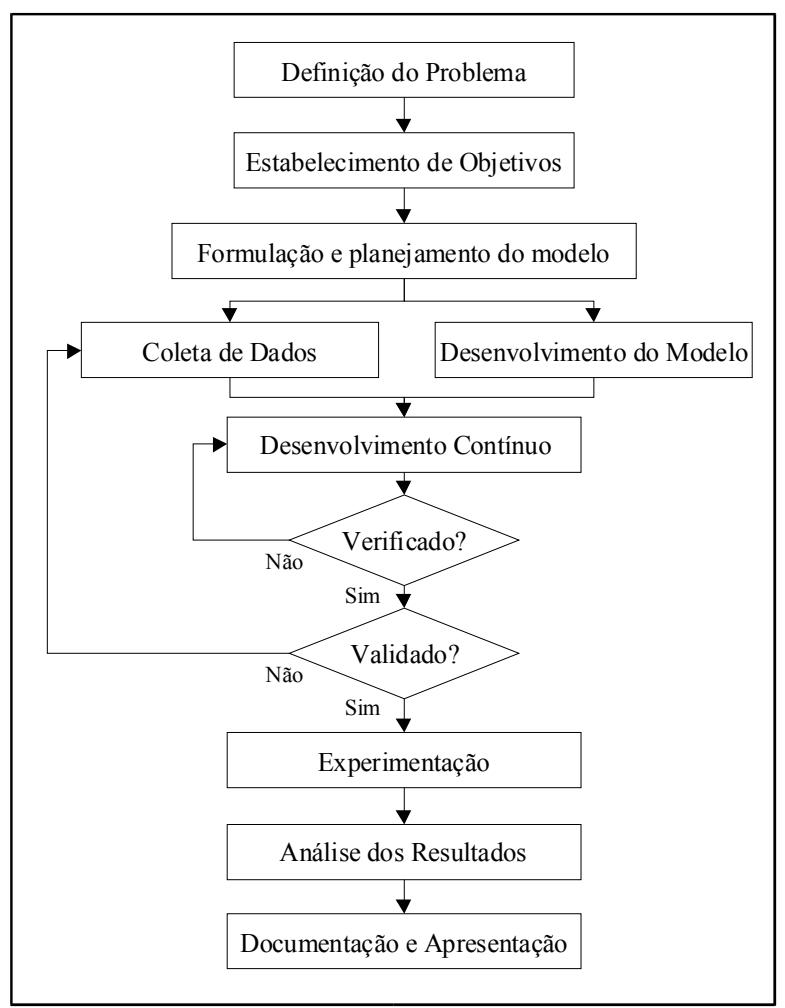

Figura 2 - Metodologia de simulação (Bateman et al., 1997) 
A fase de experimentação dos cenários dividi-se em três etapas, como pode ser visto na . Inicialmente é feita uma preparação, na qual são definidos o 'período de aquecimento', 'horizonte de simulação' e 'número de réplicas' necessárias. Depois disso, é feito o delineamento de experimentos para identificar quais variáveis de decisão têm efeito significativo sobre o custo total da cadeia. E finalmente, cada um dos cenários é simulado com diferentes parametrizações, das variáveis de decisão do modelo, na busca do menor custo da cadeia. Esta última etapa da experimentação do modelo é apresentada no próximo capítulo.

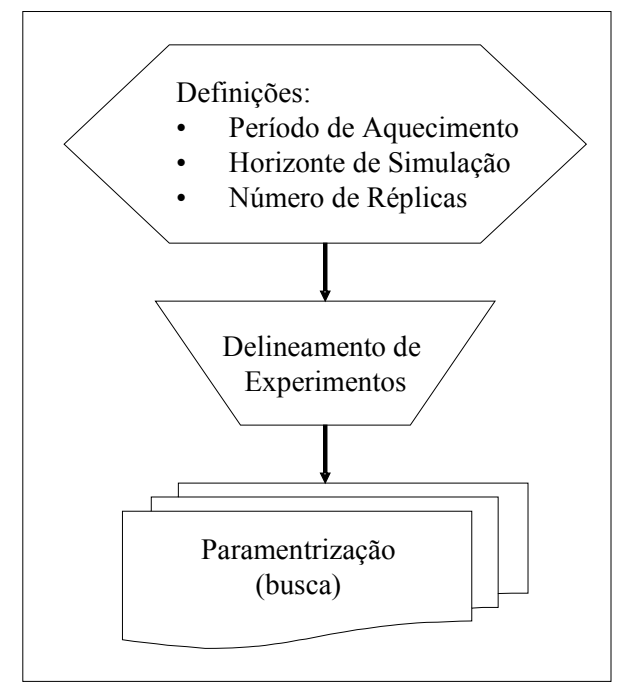

Figura 3 - Etapas da experimentação do modelo de simulação

\section{DISCUSSÃO DO CASO E PRINCIPAIS RESULTADOS}

O modelo representa o sistema real de distribuição de medicamentos de um laboratório cuja planta e armazém estão localizados no estado de São Paulo. Também foram incluídos no modelo os oitenta e um distribuidores de medicamentos representados como pontos de estocagem.

Esse modelo serve de ferramenta para teste das políticas de estocagem e auxílio na sua parametrização, considerando um ambiente em que haja cooperação e compartilhamento de informações entre o fabricante e os distribuidores. Por meio das simulações também se torna possível mensurar parte dos benefícios associados à cooperação e ao compartilhamento de informações.

O modelo de simulação tem basicamente três fluxos de materiais, como pode ser visto na Figura 4. A seguir, é apresentada a lógica de cada um dos três fluxos de materiais.

A Tabela 3 resume as regras utilizadas para coordenar o fluxo de materiais em cada um dos cenários simulados.

Tabela 3 - Regras dos fluxos de materiais

\begin{tabular}{|l|l|l|l|}
\hline & Cenário I & Cenário II & Cenário III e IV \\
\hline $\begin{array}{l}\text { FLUXO 1 } \\
\text { reposição de estoques do } \\
\text { laboratório }\end{array}$ & ponto de reposição & ponto de reposição & ponto de reposição \\
\hline $\begin{array}{l}\text { FLUXO 2 } \\
\text { reposição dos estoques dos } \\
\text { distribuidores }\end{array}$ & $\begin{array}{l}\text { representação do que } \\
\text { aconteceu no passado } \\
\text { (dados históricos) }\end{array}$ & cálculo de necessidades & $\begin{array}{l}\text { cálculo de } \\
\text { necessidades }\end{array}$ \\
\hline $\begin{array}{l}\text { FLUXO 3 } \\
\text { demanda de cada distribuidor e } \\
\text { envio dos medicamentos à } \\
\text { farmácia }\end{array}$ & $\begin{array}{l}\text { representação } \\
\text { determinística do que } \\
\text { aconteceu no passado } \\
\text { (dados históricos) }\end{array}$ & $\begin{array}{l}\text { representação } \\
\text { determinística do que } \\
\text { aconteceu no passado } \\
\text { (dados históricos) }\end{array}$ & $\begin{array}{l}\text { distribuições de } \\
\text { probabilidade } \\
\text { construídas com base } \\
\text { nos históricos }\end{array}$ \\
\hline
\end{tabular}




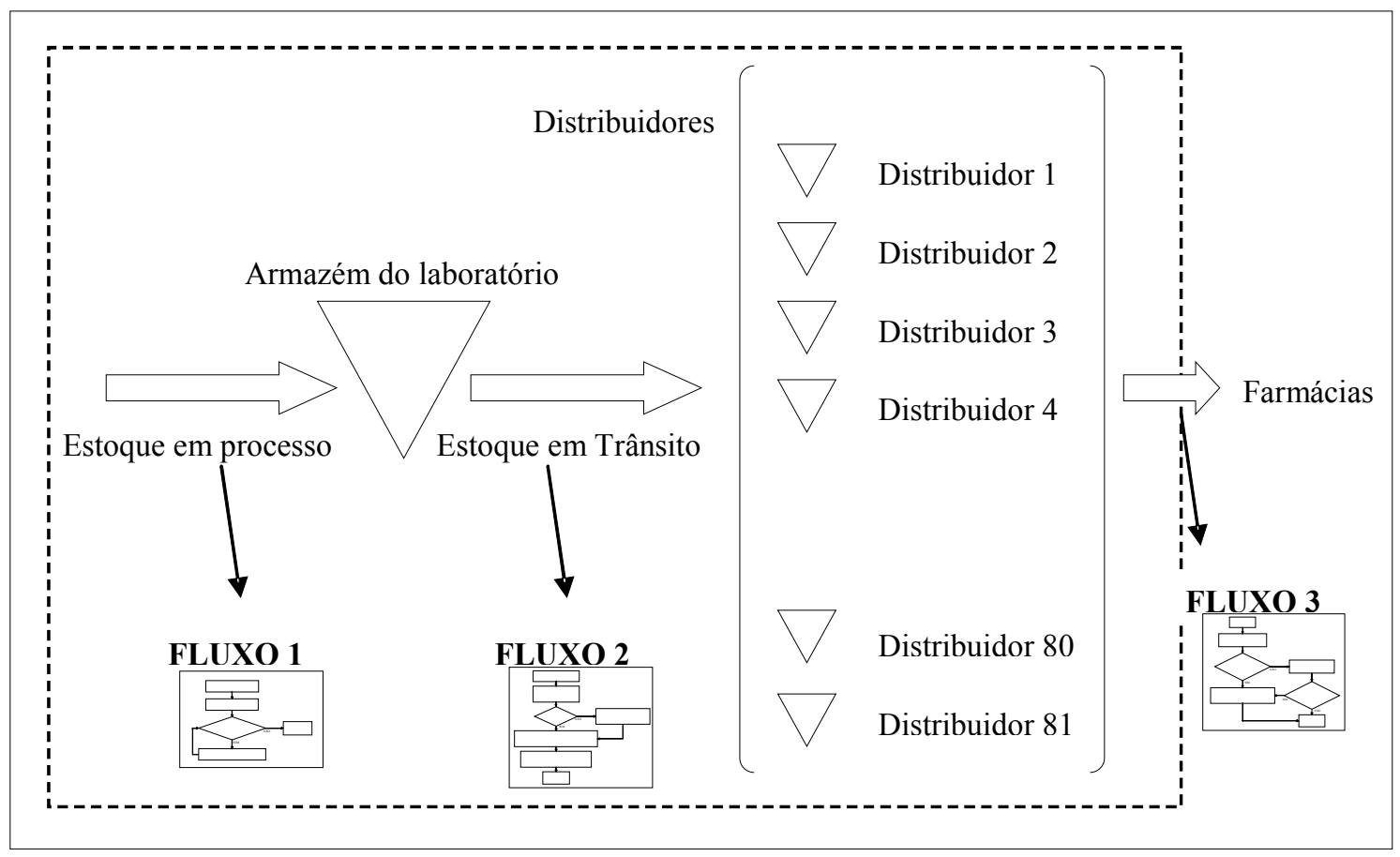

Figura 4 - Representação do sistema simulado

Na modelagem do custo total da cadeia foram consideradas as seguintes componentes:

- Custo de estoque (distribuidor)

- Custo de estoque (laboratório)

- Custo de pedido

- Custo de transporte

- Custo de entrega em atraso (laboratório)

- Custo de venda perdida (distribuidor)

Para verificar e quantificar os benefícios que a lógica de gestão dos estoques simulada tem sobre as práticas atuais adotadas pelo mercado em questão foram criados e simulados 6 cenários.

\section{Cenário I}

O primeiro cenário simulado refere-se aos dados históricos do sistema. Os resultados desse cenário serviram como base para validação do modelo, uma vez que estes foram avalizados por profissionais da empresa na qual o estudo foi realizado. O segundo objetivo deste cenário é ter seu desempenho fixado como base de comparação para os outros cenários simulados.

\section{Cenário II}

O segundo cenário simulado representa o fluxo de materiais que teria ocorrido entre o laboratório, os distribuidores e as farmácias caso uma política de cálculo de necessidades tivesse sido utilizada para a reposição dos estoques dos distribuidores. A previsão de demanda semanal de cada distribuidores foi calculada com a média móvel da sua demanda nas últimas cinqüenta e duas semanas. Considerou-se também a sazonalidade da demanda do distribuidor através do ajuste da previsão com os índices de sazonalidade semanal do ano anterior.

Outro pressuposto importante na construção deste e dos próximos cenários é a cooperação entre as empresas que fazem parte do sistema. Essa cooperação é possível com a utilização de uma ferramenta informatizada que gerencia a emissão dos pedidos.

\section{Cenário III}

O cenário III é uma réplica do segundo cenário. Entretanto, este cenário foi construído para que fosse realizada a análise do custo total da cadeia frente à variação das variáveis de decisão do modelo. Dessa forma, a demanda observada por cada um dos distribuidores, que no segundo cenário era uma variável determinística, foi substituída pela respectiva distribuição de probabilidade. 


\section{Cenário IV}

O cenário IV é uma variação do cenário III em que a previsão de vendas do laboratório é feita com base no histórico de vendas agregadas dos distribuidores. O objetivo deste cenário é confirmar e quantificar a capacidade de redução do efeito bullwhip e do custo total da cadeia por meio do compartilhamento de informações de vendas entre os distribuidores e o laboratório.

Para verificar o desempenho da política simulada frente a uma demanda com sazonalidade foram criados outros dois cenários, os cenários IIIS e IVS. O cenário IIIS é idêntico ao cenário III acrescido da componente de sazonalidade. Assim como, o cenário IVS é o cenário IV com sazonalidade.

Para definição de quais variáveis de decisão devem ser varridas pelo método de busca realizou-se um delineamento de experimentos. Foi feito um experimento fatorial completo (de dois níveis) com as variáveis de decisão do modelo.

O resultado final do delineamento, mostrou que, ao nível de significância de $90 \%$, para o cenário III as variáveis de decisão que têm influência significativa sobre o custo total da cadeia são o fator de segurança dos distribuidores e a interação entre o período de revisão dos estoques dos distribuidores e o fator de segurança dos distribuidores. Já para o cenário IV, além das variáveis significativas para o cenário III, também se mostraram importantes os efeitos provocados pelas variações do tamanho do lote de produção no laboratório e pelo período de revisão dos estoques dos distribuidores.

Apesar de significativo para a definição do custo total da cadeia no cenário IV, o 'tamanho do lote de produção no laboratório' não foi considerado como uma variável de decisão durante as simulações por três razões principais:

- A empresa foco do estudo apresentou restrições tecnológicas que impedem a mudança no tamanho do lote;

- Apesar de contemplado pelo modelo de simulação, não foi possível obter o valor do custo fixo de um lote de produção no laboratório;

- A consideração de mais essa variável de decisão aumentaria significativamente o tempo de simulação, inviabilizando a busca dos parâmetros.

Dessa forma, a parametrização da política de estoques empregada nos cenários de simulação tem as seguintes variáveis de decisão:

- Período de revisão dos estoques dos distribuidores;

- $\quad$ Fator de segurança dos distribuidores.

\section{Principais Resultados}

Os resultados a seguir referem-se à simulação da solução obtida com a simulação de menor custo total. Para o monitoramento do modelo foram acompanhadas as variáveis apresentadas na Tabela 4 e descritas a seguir:

- vendas do distribuidor: quantidade demandada pelas farmácias para todos distribuidores da cadeia;

- faltas no distribuidor: quantidade demandada pelas farmácias e indisponível nos distribuidores para entrega;

- nível de serviço (distrib): razão entre a quantidade de produtos entregues às farmácias e a quantidade de produtos demandados pelas farmácias;

- estoque médio (distrib): quantidade média de produtos em estoque considerando todos os distribuidores da cadeia;

- pedido ao laboratório: quantidade demandada pelos distribuidores para o laboratório;

- faltas no laboratório: quantidade demandada pelos distribuidores e indisponível para entrega;

- nível de serviço (lab): razão entre a quantidade de produtos entregues aos distribuidores e a quantidade de produtos demandados pelos distribuidores;

- estoque médio (lab): quantidade média de produtos em estoque no laboratório;

- número de pedidos dos distribuidores: número de pedidos recebidos pelo laboratório;

- número de ordens de produção: número de lotes de produção feitos pelo laboratório;

- quantidade entregue às farmácias: quantidade de produtos entregues às farmácias. 
Tabela 4 - Resultados físicos da simulação

\begin{tabular}{|c|c|c|c|c|c|c|}
\hline & 1 & II & III & IV & IIIS & IVS \\
\hline Vendas do Distribuidor - unidades & 1.307 .342 & 1.312 .400 & 1.343 .130 & 1.343 .136 & 1.259 .296 & 1.263 .233 \\
\hline Faltas no Distribuidor - unidades & 99.829 & 18.000 & 15.400 & 15.566 & 18.766 & 25.333 \\
\hline Nível de Serviço (distrib) & $92,4 \%$ & $98,6 \%$ & $98,9 \%$ & $98,8 \%$ & $98,5 \%$ & $98,0 \%$ \\
\hline Estoque Médio (distrib) - unidades & 438.033 & 45.321 & 40.415 & 41.654 & 77.262 & 69.634 \\
\hline Pedido ao Laboratório - unidades & 1.392 .932 & 1.319 .000 & 1.307 .000 & 1.306 .570 & 1.208 .000 & 1.207 .500 \\
\hline Faltas no Laboratório - unidades & 0 & 0 & 0 & 0 & 58.900 & 0 \\
\hline Nível de Serviço (lab) & $100 \%$ & $100 \%$ & $100 \%$ & $100 \%$ & $95,1 \%$ & $100 \%$ \\
\hline Estoque Médio (lab) - unidades & 107.274 & 96.349 & 92.861 & 94.067 & 90.017 & 96.395 \\
\hline Número de Pedidos dos Distribuidores & 831 & 8.494 & 8.874 & 8.687 & 5.860 & 5.799 \\
\hline Número de Ordens de Produção & 10 & 9 & 9 & 9 & 7 & 8 \\
\hline unidades & 1.207 .514 & 1.312 .400 & 1.327 .730 & 1.327 .570 & 1.240 .530 & 1.237 .900 \\
\hline
\end{tabular}

A redução do estoque médio dos distribuidores representa o principal efeito decorrente da transição da política de reposição dos estoques original de cada distribuidor (cenário I) para a política simulada. Os cenários II, III e IV quando comparados ao cenário I apresentam uma redução no estoque médio respectivamente de $89,7 \%, 90,8 \%$ e $90,5 \%$. Essa redução foi decorrente dos seguintes fatores:

- Periodicidade de entrega incrementada: com o período de revisão dos estoques diário, o estoque-alvo passou a ser calculado para suprir a demanda de apenas três dias (dois dias do tempo de ressuprimento e um dia do período de revisão). Isso teve como efeito a diminuição do lote médio pedido pelos distribuidores e o aumento da periodicidade de entrega.

- Método de previsão de demanda: nos cenários II, III e IV há um método formal e quantitativo de previsão de demanda para direcionar a reposição dos estoques apenas para o atendimento da demanda do período de revisão e o tempo de ressuprimento.

- Eliminação das compras especulativas: a análise dos dados de pedidos históricos dos distribuidores, que foram utilizados no cenário I, indica que parte das compras feitas por eles não tem como objetivo a simples reposição dos estoques e o atendimento da demanda até o próximo pedido. Ao longo das séries de pedidos, é possível encontrar picos referentes a transações cuja quantidade envolvida é muito superior à média histórica de pedidos do distribuidor. Esses pedidos que fogem a regra são seguidos por um período no qual o distribuidor diminui o volume de compras do produto.

Esse comportamento é motivado pelas políticas comerciais que premiam os grandes lotes de compra com descontos para o cliente e com premiações para a força de vendas. Entretanto, esse tipo de compra gera um estoque intermediário na cadeia que aumenta seu custo total e bloqueia o fluxo da informação de demanda para o laboratório. Com a utilização da política de cálculo de necessidades e o estoque-alvo, essa causa para o acúmulo de estoque é eliminada.

Como pode ser verificado pelos dados apresentados na Tabela 5 e na Figura 5, a simulação mostrou uma redução no custo total da cadeia em relação ao cenário I de $70,6 \%$ para o cenário II, de $72,1 \%$ para o cenário III e de $71,8 \%$ para o cenário IV. Esse resultado para o custo total indica que a política testada possui desempenho superior às práticas atuais da cadeia.

Tabela 5 - Resultado consolidado da simulação

\begin{tabular}{|c|c|c|c|c|c|c|c|}
\hline & $\mathrm{T}$ & $\pi$ & III & & IV & IIIS & IVS \\
\hline Custo Estoque (distrib) & $\mathrm{R} \$ 1.292 .205$ & $\mathrm{R} \$ 134.670$ & $\mathrm{R} \$ 120.094$ & $\mathrm{R} \$$ & 123.775 & $\mathrm{R} \$ 229.584$ & $\mathrm{R} \$ 206.918$ \\
\hline Custo de venda perdida (distrib) & $\begin{array}{|ll|}\mathrm{R} \$ & 327.438 \\
\end{array}$ & $\mathrm{R} \$ \quad 59.040$ & $\mathrm{R} \$ \quad 50.512$ & $\mathrm{R} \$$ & 51.058 & $\mathrm{R} \$ \quad 61.554$ & \begin{tabular}{|l|}
$\mathrm{R} \$ 83.093$ \\
\end{tabular} \\
\hline Custo Estoque (lab) & 263.589 & \begin{tabular}{|l|}
$R \$ 238.467$ \\
\end{tabular} & $\mathrm{R} \$ 229.835$ & $\mathrm{R} \$$ & 232.818 & $\mathrm{R} \$ 222.795$ & $\mathrm{R} \$ 238.580$ \\
\hline Custo de entrega com atraso (lab) & $\mathrm{R} \$$ & $\mathrm{R} \$$ & $\mathrm{R} \$$ & $\mathrm{R} \$$ & & \begin{tabular}{|l|l}
$\mathrm{R} \$$ & 16.877 \\
\end{tabular} & $\mathrm{R} \$$ \\
\hline Custo de Pedidos (distrib) & 11.422 & $\mathrm{R} \$ \quad 10.816$ & $\begin{array}{ll}\mathrm{R} \$ & 10.717 \\
\end{array}$ & $\mathrm{R} \$$ & 10.713 & 9.907 & 9.901 \\
\hline Custo de Transporte & 209.186 & $\mathrm{R} \$ 175.300$ & $\mathrm{R} \$ 174.829$ & $\mathrm{R} \$$ & 174.320 & $\mathrm{R} \$ 155.823$ & $\mathrm{R} \$ 155.615$ \\
\hline Custo Total da Cadeia & $\mathrm{R} \$$ & $\bar{R}$ & 98 & $\mathrm{R} \$$ & 592.684 & 5.540 & $\mathrm{R} \$ 694.107$ \\
\hline
\end{tabular}

Já na comparação do custo total da cadeia entre os cenários III (R \$ 585.987) e IV (R \$ 592.684), se forem consideradas as semi-amplitudes dos intervalos de confiança de R \$ 13.353 para o cenário III e R 11.271 para o cenário IV, não se pode afirmar que exista diferença significativa entre esses dois cenários.

A redução do custo total para os cenários II, III e IV ocorreu sobretudo pela redução do custo de estoque e de vendas perdidas dos distribuidores. Isso é conseqüência da melhor gestão dos estoques que proporcionou a melhoria simultânea dos indicadores de nível de serviço e estoque médio do sistema.

Para os custos totais dos cenários com sazonalidade IIIS (R\$ 696.540) e IVS (R\$ 694.107), cujas semiamplitudes dos intervalos de confiança são respectivamente $\mathrm{R} \$ 25.000$ e $\mathrm{R} \$ 25.900$, não se pode afirmar que exista diferença significativa entre esses dois cenários. Ou seja, para os cenários com sazonalidade, 
também não há ganho com o compartilhamento das informações de vendas dos distribuidores com o laboratório.

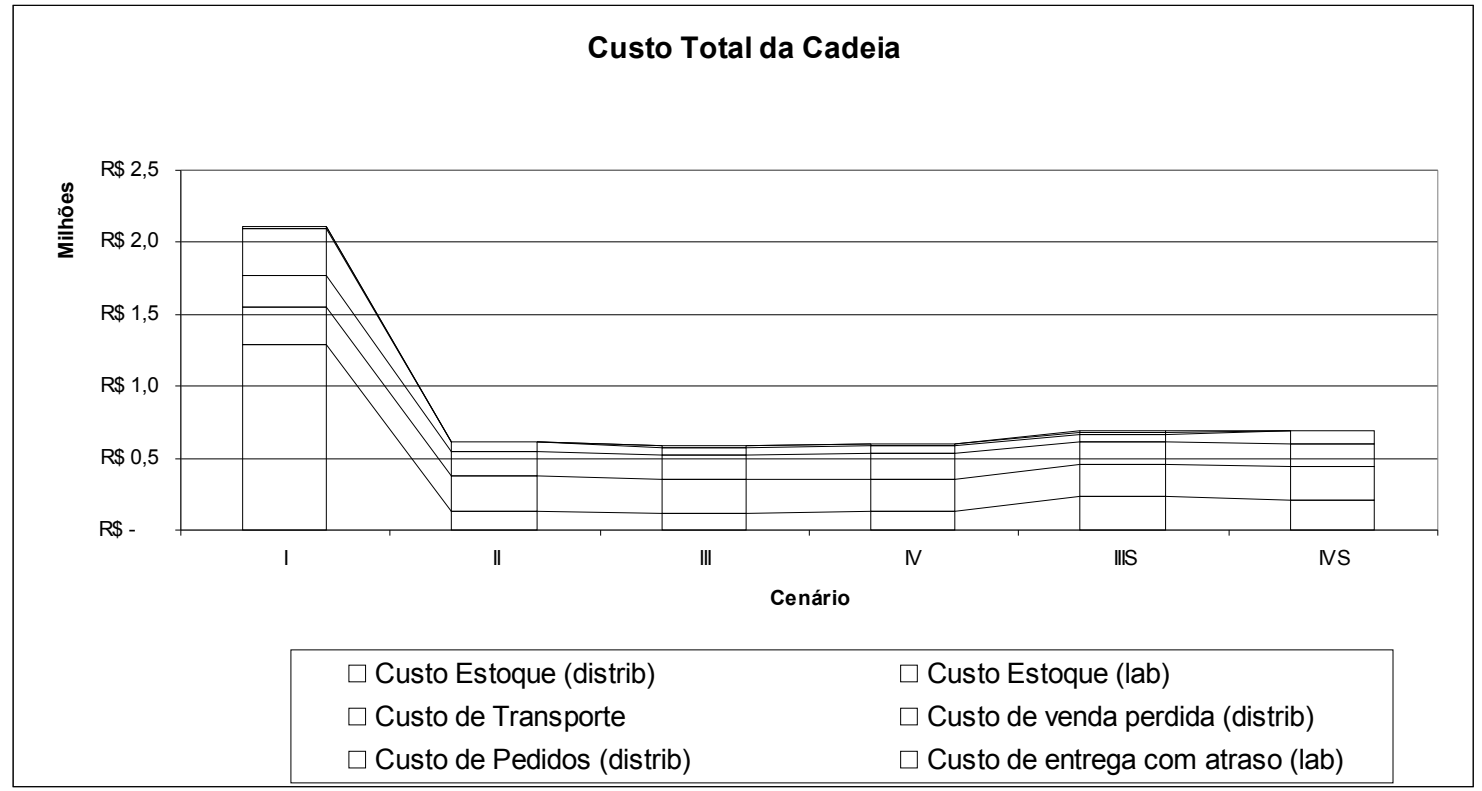

Figura 5 - Variação no custo total da cadeia

\section{CONSIDERAÇÕES FINAIS}

A Figura 6 apresenta a comparação entre o nível de serviço e custo total das soluções de cada cenário simulado em relação ao cenário I, que representa o fluxo histórico da cadeia. Por meio desse quadro é possível verificar a significativa diminuição no custo total da cadeia e aumento no nível de serviço.

Os principais fatores que levaram à redução do custo total da cadeia foram a diminuição do estoque médio dos distribuidores e o próprio aumento do nível de serviço que reduziu o custo de vendas perdidas. A melhoria simultânea do nível de serviço e do nível de estoques da cadeia mostra que a política simulada mudou o dilema (trade-off) que interliga essas duas características de desempenho do sistema de forma antagônica. Dessa forma, pode-se afirmar que o uso da política de estoques testada insere uma melhoria na tecnologia de gestão do fluxo de materiais na cadeia de distribuição.

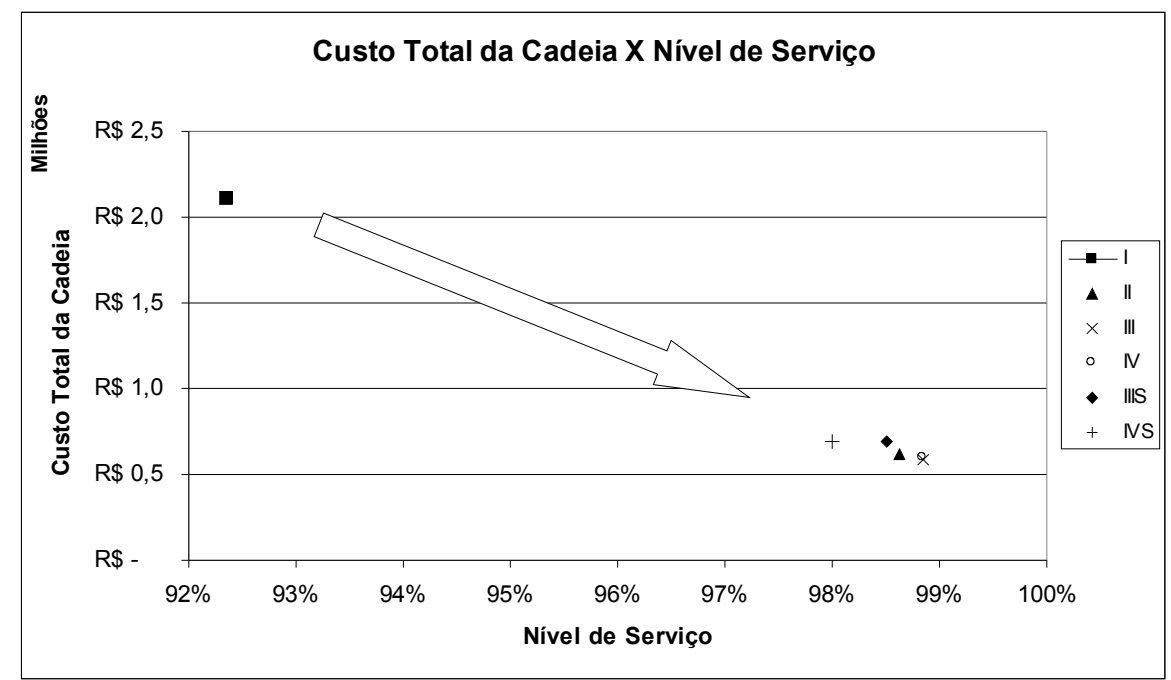

Figura 6 - Custo total da cadeia X Nível de serviço

Contudo, esperava-se um desempenho superior dos cenários IV e IVS em relação aos cenários III e IIIS respectivamente, pois nos cenários IV e IVS as previsões de vendas do laboratório foram feitas com os dados relativos às vendas dos distribuidores e não de seus pedidos. Dessa forma, previa-se que o estoque médio do laboratório pudesse ser reduzido nestes dois cenários. 
Entretanto, devido ao tamanho do lote de produção (aproximadamente igual à quantidade pedida ao laboratório em um mês), esse benefício não pode ser concretizado pelo sistema. Além disso, como a parametrização dos cenários levou a revisão mais freqüente dos estoques, a diferença entre as curvas de pedidos e vendas dos distribuidores foi praticamente eliminada. Dessa forma, pode-se concluir que, dependendo da política de reposição dos estoques empregada e da sua parametrização, os resultados apontados na pesquisa podem ser obtidos sem a necessidade de compartilhamento das informações de estoques e vendas do distribuidor com o laboratório.

No quadro apresentado na Tabela 6 , há um resumo dos resultados da simulação frente às dimensões de análise. As dimensões de análise representam as variáveis de decisão do modelo de simulação e as hipóteses estudadas nos diferentes cenários.

Tabela 6 - Relacionamento entre as dimensões de análise e os custos da cadeia

\begin{tabular}{|c|c|c|c|c|c|}
\hline $\begin{array}{c}\text { Diminuição } \\
\text { PerRev }\end{array}$ & $\begin{array}{c}\text { Diminuição } \\
\text { FS distr }\end{array}$ & $\begin{array}{l}\text { Diminuição } \\
\text { LotProd }\end{array}$ & Cooperação & $\begin{array}{l}\text { Comparti- } \\
\text { lhamento }\end{array}$ & \\
\hline$\nabla \nabla \nabla$ & $\boldsymbol{\nabla}$ & 一 & $\nabla \nabla \nabla$ & 一 & Custo de estoque (distrib) \\
\hline - & - & $\nabla \nabla \nabla$ & & - & Custo de estoque (lab) \\
\hline - & - & - & - & - & Custo de pedido \\
\hline$\Delta$ & - & - & $\boldsymbol{\Delta}$ & - & Custo de transporte \\
\hline - & - & $\boldsymbol{\Delta}$ & - & $\nabla$ & Custo de entrega em atraso (lab) \\
\hline $\mathbf{\Delta}$ & $\Delta \mathbf{\Delta} \mathbf{A}$ & - & $\boldsymbol{\nabla}$ & - & Custo de venda perdida (distrib) \\
\hline & & $\mathbf{\Delta}(?)$ & & & Custo de prep. da linha (setup) \\
\hline$\nabla \nabla \nabla$ & $\Delta \mathbf{\Delta} \boldsymbol{\Delta}$ & $(?)$ & $\nabla \nabla \nabla$ & - & Custo total da cadeia \\
\hline$\nabla \nabla \nabla$ & - & & $\nabla \nabla \nabla$ & $\boldsymbol{\nabla}$ & $\mathrm{I}_{\text {chicote }}$ \\
\hline
\end{tabular}

Legenda:

PerRev - Período de revisão dos distribuidores

FS distr - Fator de segurança dos distribuidores

LotProd - Lote de produção no laboratório

Cooperação - Existência de Cooperação entre os elos da cadeia de distribuição

Compartilhamento - Compartilhamento de informações entre as empresas da cadeia
$\Delta$ - Aumento relativamente pequeno
$\Delta \boldsymbol{\Delta} \mathbf{\Delta}-$ Aumento relativamente grande
$\boldsymbol{\nabla}$ - Diminuição relativamente pequena
$\boldsymbol{\nabla} \nabla$ - Diminuição relativamente grande
- - Nenhum efeito observado

A diminuição do período de revisão dos estoques dos distribuidores e o conseqüente aumento na periodicidade de entrega, ocasionaram aumento relativamente pequeno no custo de transporte e de venda perdida, e a redução relativamente grande no custo de estoque dos distribuidores.

A diminuição do fator de segurança dos distribuidores teve como conseqüência uma redução relativamente pequena no custo de estoque dos distribuidores versus um aumento relativamente grande no custo de vendas perdidas, devido à característica de lucratividade da indústria em questão.

A diminuição no tamanho do lote de produção apresentou redução relativamente grande no custo de estoque do laboratório. Essa alteração tem como conseqüência o aumento do 'custo de preparação da linha de produção'. Entretanto, a quantificação desse aumento não foi feita nesse estudo, pois não estava disponível o 'custo unitário de preparação da linha de produção'.

Parte significativa da redução no custo total da cadeia baseia-se na eliminação da prática de compras especulativas. Para que o ganho relacionado à sua eliminação possa ser efetivamente obtido pela cadeia de distribuição, é preciso que deixe de existir motivações para os distribuidores fazerem esse tipo de compra. Esse é um dos pressupostos para as conclusões da pesquisa.

Os cenários que exploraram o compartilhamento de informações não apresentaram benefício significativo. Com relação ao compartilhamento de informações, observou-se apenas uma redução relativamente pequena no custo de entrega com atraso, para os cenários com sazonalidade. 


\section{REFERENCIAS BIBLIOGRÁFICAS}

BALLOU, R. Business Logistics Management: planning, organizing, and controlling the supply chain. 4. ed. Londres: Prentice Hall, 1998. 696 p.

Logística Empresarial: transportes, administração de materiais, distribuição física. 11. ed. São Paulo: Atlas, 1987. 392 p.

BATEMAN, R. et. al. A Simulação Aprimorando Sistemas. 1. ed. Orem, 1997. 146 p.

BOWDEN, R; HALL, J. Simulation Optimization Research and Development. Proceedings of the 1998 Winter Simulation Conference. 1998. p 1693-1698.

BOWERSOX, D; CLOSS, D. Logistical Management: The Integrated Supply Chain Process. Mc Graw Hill, Singapura, 1996. $752 \mathrm{p}$.

FORRESTER, J. Industrial Dynamics. Harvard Business Review. Boston, No. 36, p. 37-52, JulhoAgosto 1958.

LEE, H.L.; Billington C. Managing Supply Chain Inventory: Pitfalls and Opportunities. Sloan Management Review, Cambridge, Spring, p.65-73, 1992.

LEE, H.L; PADMANABHAN, V.; WHANG,S. Information Distortion in a Supply Chain: The Bullwhip Effect, Management Science, vol.43, No.4, p.546-558, 1997.

The Bullwhip effect in supply chains. Sloan Management Review, Cambridge, Spring, p.93-102, 1997b.

LEE, H.L.; WHANG, S. Information Sharing in a Supply Chain. Stanford University, Department of Industrial Engineering and Engineering Management, Stanford, 1998.

ROBENSON, J.; COPACINO, W; HOWE, R. The Logistics Handbook. Nova Iorque: Free Press, 1994. $954 \mathrm{p}$.

SIMCHI-LEVI, D.; KAMINSKY, P.; SIMCHI-LEVI, E. Designing and Managing the Supply Chain: Concepts, Strategies, and Case Studies. Boston: McGraw-Hill, 2000. 321 p.

SIMCHI-LEVI, D. Coordinating Production, Distribution and Transportation Decisions in the Supply Chain. In: 1998 NSF Workshop On Supply Chain Management In Electronic Commerce, 1998, Gainesville. Anais eletronicos... Disponível em: $<$ http://www.ise.ufl.edu/Supplychain / done/Day3/Levi/>, Acesso em: 28 nov. 2000.

TAYUR, S.; GANESHAN, R; MAGAZINE, M. Quantitative Models for Supply Chain Management. Dordrecht Hardbound: Kluwer Academic Publishers, 1998. 896 p. 